\title{
Reduced Krüppel-Like Factor 2 Aggravates Glomerular Endothelial Cell Injury and Kidney Disease in Mice with Unilateral Nephrectomy
}

Fang Zhong, ${ }^{* \dagger}$ Sandeep K. Mallipattu, ${ }^{\ddagger}$ Chelsea Estrada, ${ }^{\ddagger}$ Madhav Menon, ${ }^{*}$ Fadi Salem, ${ }^{\S}$ Mukesh K. Jain, ${ }^{\mp}$ Hongyu Chen, Yongjun Wang, ${ }^{\dagger}$ Kyung Lee, ${ }^{*}$ and John C. He ${ }^{*} \|$

From the Departments of Medicine/Nephrology* and Pathology, ${ }^{\complement}$ Icahn School of Medicine at Mount Sinai, New York, New York; the Department of Nephrology, ${ }^{\dagger}$ Hang Zhou Hospital of Traditional Chinese Medicine, Zhejiang Chinese Medical University, Hangzhou, China; the Division of Nephrology, Department of Medicine, Stony Brook University, Stony Brook, New York; the Department of Medicine, Case Cardiovascular Institute Research Institute, Case Western Reserve University, Cleveland, Ohio; and the Renal Section," James J. Peters Veterans Affairs Medical Center, Bronx, New York

Accepted for publication March 29, 2016.

Address correspondence to John C. He, M.D., Division of Nephrology, Box 1243, Mount Sinai School of Medicine, One Gustave L. Levy Place, New York, NY 10029. E-mail: cijiang.he@mssm.edu.

\begin{abstract}
Loss of functional nephrons induces compensatory glomerular hyperfiltration and hypertrophy, leading to the progression of chronic kidney disease. Krüppel-like factor 2 (KLF2), a shear-stress-inducible transcription factor, confers protection against endothelial injury. Because glomerular hyperfiltration is associated with shear stress, we hypothesized that KLF2 may be an important factor in the compensatory response to unilateral nephrectomy (UNX). To test this hypothesis, endothelial cell-specific Klf2 heterozygous knockout mice (KO) and their wild-type littermate control (WT) underwent either UNX or sham-operation. WT-UNX mice developed compensatory renal hypertrophy as expected, whereas KO-UNX mice did not. KO-UNX mice exhibited higher blood pressure, reduced glomerular filtration rate, and significant increase in proteinuria and glomerulosclerosis compared to WT-UNX. Expression of endothelial nitric oxide synthase (official name Nos3), a known transcriptional target gene of KLF2, was significantly reduced and dysregulation of other endothelial genes was also observed in the glomeruli of KO-UNX when compared to WT-UNX and sham-operated mice. Furthermore, both podocyte number and expression of podocyte markers were also significantly reduced in KO-UNX glomeruli, indicating a potential cross talk between glomerular endothelial cells and podocytes. Finally, decreased renal expression of KLF2 in nephrectomy patients was associated with the progression of kidney disease. Taken together, our data demonstrate a protective role of KLF2 against glomerular endothelial cell injury and progression of chronic kidney disease in the model of compensatory renal hypertrophy. (Am J Pathol 2016, 186: 2021-2031; http://dx.doi.org/10.1016/j.ajpath.2016.03.018)
\end{abstract}

The incidence of chronic kidney disease (CKD) and endstage renal disease is rapidly increasing in the United States. ${ }^{1}$ A reduction in the number of functional nephrons, whether induced genetically, surgically, or by acquired renal disease, contributes to the progression of CKD, likely secondary to glomerular hyperfiltration and hypertrophy. ${ }^{2}$ However, the precise mechanism that mediates the progression of CKD in these patients remains unclear.

Krüppel-like factors (KLFs) are a subfamily of 17 DNAbinding transcriptional regulators that are involved in a broad range of cellular processes, such as cell differentiation, angiogenesis, erythropoiesis, and immune regulation. $^{3-9}$ Among KLF proteins, KLF2 is a key regulator of vascular hemodynamic forces in vivo ${ }^{10}$ and mediates flowdependent phenotype in endothelial cells. ${ }^{3,11}$ KLF2 has been shown to be essential in maintenance of endothelial integrity in adult mice ${ }^{12}$ as well as in mouse embryonic vasculature. $^{13}$ KLF2 inhibits vascular endothelial growth factor (VEGF)-A-mediated angiogenesis ${ }^{14}$ and regulates endothelial thrombotic function. ${ }^{15}$ In addition, KLF2 exhibits anti-inflammatory effects in endothelial cells,

Supported by Chinese 973 fund 2012CB517601 (J.C.H.), VA Merit Award (J.C.H.), and NIH 1R01DK078897, 1R01DK088541, and P01-DK56492 (J.C.H.) and K08DK102519 (S.K.M.).

Disclosures: None declared. 
thereby protecting cells from injury in response to cell stress. ${ }^{16}$

Although the role and function of KLF2 has been extensively studied in endothelial cell biology, studies examining its role in kidney disease have been sparse in comparison. It has been shown that chronic exposure to laminar shear stress induces KLF2 expression in glomerular endothelial cells. ${ }^{17}$ Our recent study suggests that KLF2 is up-regulated by both high glucose and insulin and it protects against endothelial cell injury in early diabetic nephropathy.$^{18}$ In addition to high glucose and insulin, we hypothesized that the increased shear stress in glomerular endothelial cells during the early stages of diabetic nephropathy would contribute to the up-regulation of KLF2 expression. We were previously unable to address this hypothesis in the diabetic kidney because of the effects of high glucose and insulin on KLF2 expression. Therefore, in the present study, we have used the unilateral nephrectomy model, in which glomerular hyperfiltration and hypertrophy is the major contributor to the development and progression of CKD. Our results demonstrate that KLF2 expression is induced by glomerular hyperfiltration in the early stage of CKD and confers protection against endothelial cell dysfunction, thereby attenuating the progression of CKD in mice with unilateral nephrectomy.

\section{Materials and Methods}

\section{Generation of Conditional Klf2 Mice}

$K l f 2^{f l+}$ mice were generated by deletion of exons 2 and 3 and provided by Dr. Mukesh Jain, a collaborator of this study. Klf $2^{f /+}$ mice in C57BL/6 background were viable, fertile, and born in the mendelian ratios as expected. $V E C$ Cre transgenic animals were purchased from Jackson Research Laboratories (Bar Harbor, ME). Endothelial cell-specific heterozygous Klf2 knockout mice (KO) were generated as described in Results.

\section{UNX Model}

All mice used in this study were on a congenic C57BL/6 background. Eight-week-old male mice weighing 23 to $28 \mathrm{~g}$ were used in the study. Wild-type (WT) and KO mice were randomly divided into two groups ( $n=6$ per group) for unilateral nephrectomy (UNX) of right kidney and shamoperation. UNX was performed in anesthetized mice through dorsal skin. An incision was made in the left psoas muscle wall. The left renal pedicle was identified and ligated, followed by total removal of left kidney. Then, the left psoas muscle and overlying skin were sutured and mice were warmed and monitored until full recovery from anesthesia. Sham-operated mice had the left renal pedicles manipulated without ligation. Urine, serum, and body weight were checked biweekly. Urine and serum were collected, and blood pressure and body and kidney weight were recorded at 12 weeks post-UNX or sham operation before animals were sacrificed. All protocols were approved by the Animal Care Committee at Icahn School of Medicine at Mount Sinai (New York, NY).

\section{Measurement of Urine Albumin and Creatinine}

Urine albumin was quantified by enzyme-linked immunosorbent assay using a kit from Bethyl Laboratories, Inc. (Houston, TX). Urine creatinine levels were measured in the same samples using QuantiChrom creatinine assay kit (DICT-500) (BioAssay Systems, Hayward, CA) according to the manufacturer's instruction. The urine albumin excretion rate was expressed as the ratio of albumin/creatinine. Twelve hours urine collection was done by using a metabolic cage.

\section{Blood Pressure Monitoring}

Blood pressure was measured using the CODA programmable noninvasive tail-cuff sphygmomanometer (Kent Scientific, Torrington, CT) on conscious mice as described. ${ }^{19}$ Mice were initially subjected to acclimation period of five cycles before blood pressure assessment. Subsequently, systolic blood pressure was measured in each mouse for 60 continuous cycles and an average of systolic blood pressure was quantified as previously described. ${ }^{19}$

\section{Measurement of Glomerular Filtration Rate}

Glomerular filtration rate (GFR) in mice was determined by using the clearance of fluorescein isothiocyanate-inulin as described in the AMDCC and Rieg. ${ }^{20}$ Briefly, 10-week-old anesthetized mice were injected retroorbitally with dialyzed $5 \%$ fluorescein isothiocyanate-inulin. Blood was collected from the tail at $3,5,7,10,15,35,56$, and 75 minutes after injection. Separated serum was buffered in $0.5 \mathrm{~mol} / \mathrm{L}$ HEPES, pH 7.4, and fluorescence was measured as described previously. ${ }^{20}$ GFR was calculated using the twocompartment clearance model with the following equation:

$$
\mathrm{GFR}=\frac{\mathrm{I}}{\frac{\mathrm{A}}{\alpha}+\frac{\mathrm{B}}{\beta}}
$$

where I is the total amount of fluorescein isothiocyanateinulin delivered in the bolus injection retroorbitally, $\mathrm{A}$ is the $y$-intercept of the rapid phase of elimination, B is the $y$-intercept of the slow phase of elimination, $\alpha$ is the decay constant for elimination, and $\beta$ is the decay constant for distribution.

\section{Kidney Histology}

Kidneys were removed and fixed with $4 \%$ paraformaldehyde for 48 hours at $4^{\circ} \mathrm{C}$. The $4 \mu \mathrm{m}$ sections were cut from paraffin-embedded kidney tissues. Sections were stained with periodic acid-Schiff for histology analysis. Assessment of the mesangial and glomerular cross-sectional 
areas was performed by pixel counts on a minimum of 10 glomeruli per section in a blinded manner (F.Z.), under $\times 400$ magnification (Zeiss AX10 microscope; Carl Zeiss Canada Ltd, Toronto, ON, Canada).

\section{Isolation of Glomeruli from Mice for RNA Extraction}

Mouse glomeruli were isolated as described. ${ }^{21}$ Briefly, animals were perfused with Hanks' buffered salt solution containing $2.5 \mathrm{mg} / \mathrm{mL}$ iron oxide and $1 \%$ bovine serum albumin. At the end of perfusion, kidneys were removed, decapsulated, minced into $1-\mathrm{mm}^{3}$ pieces, and digested in Hanks' buffered salt solution containing $1 \mathrm{mg} / \mathrm{mL}$ collagenase A and $100 \mathrm{U} / \mathrm{mL}$ deoxyribonuclease I. Digested tissue was then passed through a $100-\mu \mathrm{m}$ cell strainer and collected by centrifugation. The pellet was resuspended in $2 \mathrm{~mL}$ of Hanks' buffered salt solution, and glomeruli were collected using a magnet. The purity of glomeruli was verified under microscopy. Total RNA was isolated from kidney glomeruli of mice using TRIzol (Invitrogen, Carlsbad CA).

\section{Real-Time PCR}

Total RNA was extracted by using TRIzol (Invitrogen). First strand cDNA was prepared from total RNA $(2.0 \mu \mathrm{g})$ using the Superscript III first strand synthesis kit (Invitrogen) and $1 \mu \mathrm{L}$ cDNA was amplified in triplicate using SYBR GreenER qPCR Supermix on an ABI PRISM 7900HT (Applied Biosystems, Foster City, CA). The primer sequences are listed in Table 1. ABI 7500 analysis software version 2.0.6 (Applied Biosystems) was used to determine crossing points using the second derivative method. Data were normalized to housekeeping genes (glyceraldehyde-3phosphate dehydrogenase) and presented as fold increase compared with RNA isolated from WT animals using the $2^{-\Delta \Delta \mathrm{C}_{\mathrm{T}}}$ method.

\section{Immunofluorescence}

Kidney sections from these mice were prepared in an identical manner. Immunostaining was performed using rabbit antisynaptopodin (Fitzgerald Industries International, Acton, MA), rabbit anti-podocin (a gift from Dr. Peter Mundel,
Massachusetts General Hospital, Boston MA), and mouse anti-WT1 antibodies (Santa Cruz Biotechnology, Dallas, TX). After washing, sections were incubated with a fluorophorelinked secondary antibody (Alexa Fluor 488 anti-rabbit IgG and Alexa Fluor 568 anti-mouse IgG from Invitrogen). After staining, slides were mounted in Aqua Poly/Mount (Polysciences Inc., Warrington, PA) and photographed under an AxioVision IIe microscope with a digital camera.

\section{Immunohistochemistry}

Archival human biopsy specimens of healthy donor nephrectomies and diabetic nephropathy were collected at Icahn School of Medicine at Mount Sinai under a protocol approved by the Institutional Review Board. Archival human nephrectomy samples were obtained from SUNY at Stony Brook under a protocol approved by the Institutional Review Board. These patients underwent nephrectomy because of renal tumor and the normal kidney tissues outside of tumor were used for immunostaining. Specimens were initially baked for 20 minutes in $55^{\circ} \mathrm{C}$ to $60^{\circ} \mathrm{C}$ oven and then processed as described previously. ${ }^{18}$ Briefly formalin-fixed and paraffin-embedded sections were deparaffinized, and endogenous peroxidase was inactivated with hydrogen peroxide. Sections were then blocked in $2 \%$ goat serum in phosphatebuffered saline for 1 hour at room temperature and then incubated with a rabbit anti-KLF2 antibody (1:1000, GenScript, Piscataway, NJ) at $4^{\circ} \mathrm{C}$ overnight. The next day, sections were washed three times with phosphate-buffered saline and then incubated with secondary antibody for 30 minutes. Positive staining was revealed by peroxidase-labeled streptavidin and diaminobenzidine substrate. The control included a section stained with only secondary antibody.

\section{Quantification of Immunostaining}

After sections were stained with anti-KLF2 antibody, negatives were digitized, and images with a final magnitude of approximately $\times 400$ were obtained. ImageJ software version 1.26t (NIH, Bethesda, MD) was used to measure the level of immunostaining in the glomeruli. First, the images were converted to 8-bit grayscale. Next, the glomerular region was selected for measurement of area and integrated

Table 1 Primers for Real-Time PCR

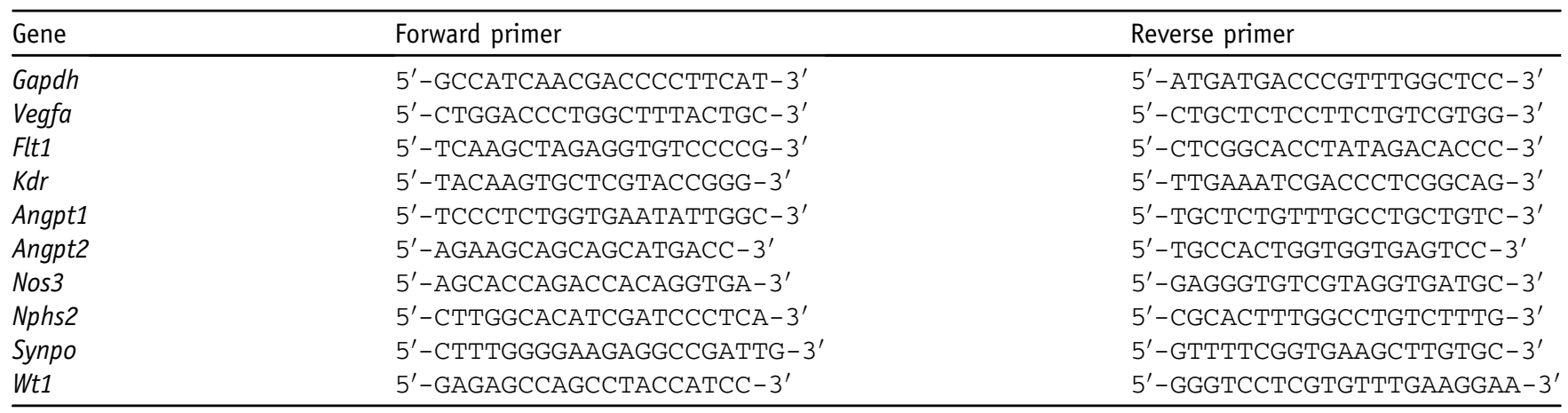


density. Next, the background intensity was measured by selecting three distinct areas in the background with no staining. The corrected optical density (COD) was determined as: $\mathrm{COD}=I D-(A \times M G V)$, where $I D$ is the integrated density of the selected glomerular region, $A$ is the area of the selected glomerular region, and $M G V$ is the mean gray value of the background readings. ${ }^{22}$

\section{Western Blot}

Mouse glomeruli were isolated as described ${ }^{21}$ then were homogenized in lysis buffer containing protease inhibitor cocktail. Equal amounts of protein samples were electrophoretically separated on SDS-polyacrylamide gel, transferred to polyvinylidene difluoride membranes (EMD Millipore, Billerica, MA), and probed with primary antibodies. Membranes were then washed with phosphatebuffered saline with Tween 20 and incubated with a secondary antibody (horseradish peroxidase conjugated antibodies to mouse $\operatorname{IgG}$ or to rabbit $\operatorname{IgG}$ ). Blots were developed with the enhanced chemiluminescence system. Densitometry analysis for quantification was performed as described previously. ${ }^{23}$

\section{Electron Microscopy}

Tissues were fixed in $2.5 \%$ glutaraldehyde with $0.1 \mathrm{~mol} / \mathrm{L}$ sodium cacodylate (pH 7.4) for 72 hours at $40^{\circ} \mathrm{C}$. Samples were further incubated with $2 \%$ osmium tetroxide and 0.1 $\mathrm{mol} / \mathrm{L}$ sodium cacodylate $(\mathrm{pH} 7.4)$ for 1 hour at $40^{\circ} \mathrm{C}$. Ultrathin sections were stained with lead citrate and uranyl acetate and were viewed on a Hitachi H7650 microscope. Briefly, negatives were digitized, and images with a final magnitude of approximately $\times 10,000$ were obtained. ImageJ software version $1.26 \mathrm{t}$ ( $r$ sb.info.nih.gov) was used to measure the length of the peripheral glomerular basement membrane (GBM), and the number of slit pores overlying this GBM length was counted. The arithmetic mean of the foot process width $\left(W_{\mathrm{FP}}\right)$ was calculated as:

$$
W_{\mathrm{FP}}=\frac{\pi}{4} \times \frac{\sum \mathrm{GBM} \text { LENGTH }}{\sum \text { silts }}
$$

where $\Sigma$ slits indicates the total number of slits counted; $\Sigma$ GBM LENGTH indicates the total GBM length measured in one glomerulus, and $\pi / 4$ is the correction factor for the random orientation by which the foot processes were sectioned. $^{24}$

\section{Statistical Analysis}

Data were expressed as means \pm SEM. The unpaired $t$-test was used to analyze data between two groups. The analysis of variance, followed by Bonferroni correction, was used when more than two groups were present. All experiments were repeated at least three times, and representative experiments are shown. Statistical significance was set at $P<0.05$.

\section{Results}

\section{Generation of Endothelial Cell-Specific Klf2 Heterozygous Knockout Mice}

Because there are no glomerular endothelial cell-specific Cre mice available, we generated global endothelial cellspecific Klf2 knockout mice by crossing the Klf2 floxed mice $^{25}$ with VE-cadherin promoter driven Cre transgenic mice (VEC-Cre). Because Klf2 $2^{\mathrm{f} / \mathrm{fl}} ; \mathrm{VEC}-\mathrm{Cre}$ homozygous mice die embryonically, ${ }^{25} \mathrm{Klf} 2{ }^{\mathrm{f} / /+}$;VEC-Cre heterozygous knockout and Klf $2^{+/+}$;VEC-Cre wild-type littermate control mice (hereafter referred as KO and WT, respectively) were used in our study. KO mice were viable, fertile, and grossly normal. Our previous study showed a 50\% reduction of Klf2 mRNA expression in isolated glomerular endothelial cells from KO kidneys as compared to those from WT kidneys. ${ }^{18}$ In addition, at baseline, KO mice did not exhibit albuminuria, glomerular injury, or tubulointerstitial disease when examined at 6 and 12 months of age (data not shown).

\section{Reduced KLF2 Expression Results in Significant Proteinuria and Kidney Injury after UNX}

Eight-week-old WT and KO mice were subject to either sham-operation or UNX and sacrificed 12 weeks post-UNX. UNX resulted in small reduction in body weight of both WT and KO mice over the course of 12 weeks as compared to sham-operated mice, with no significant differences observed between WT-UNX and KO-UNX mice (Figure 1A). As anticipated, WT-UNX developed compensatory kidney hypertrophy, as measured by an increase in kidney/body weight ratio (Figure 1B). Interestingly, this compensatory mechanism was not observed in KO-UNX mice. Furthermore, KO-UNX mice exhibited a marked reduction in GFR (Figure 1C) and a significant increase in systolic blood pressure at 12 weeks post-UNX (Figure 1D). The observed increase in blood pressure is likely secondary to declining renal function in KO-UNX mice. Consistent with declining renal function, from 4 weeks post-UNX, KO-UNX mice demonstrated a significant increase in albuminuria as compared to sham-operated mice (Figure 2A), indicative of increased glomerular injury. Although the WT-UNX mice had a slight trend of increased albuminuria, statistical significance was not reached when compared to sham-operated mice. At 12 weeks post-UNX, KO-UNX mice exhibited a significant increase in albuminuria as compared to all other groups. The increase in albuminuria was also confirmed by measuring of total albumin in a 12-hour urine collection at 12 weeks post-UNX (Figure 2B).

Histological analysis using periodic acid-Schiff staining revealed a significant increase in glomerular volume in WTUNX mice but not in KO-UNX mice, consistent with a failure of compensation in KO-UNX kidneys as observed above. In addition, glomerular mesangial matrix area in KO-UNX mice was markedly increased as compared to all 


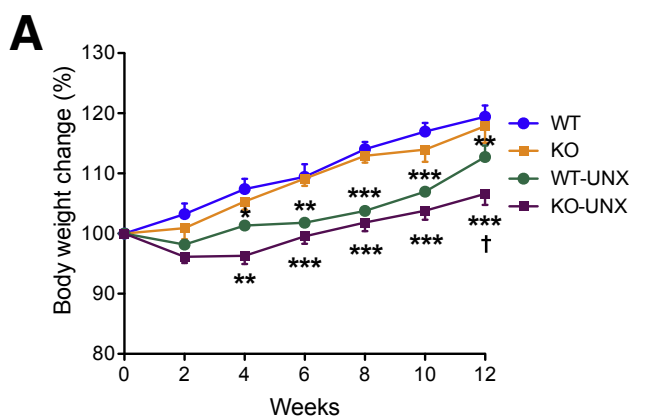

C

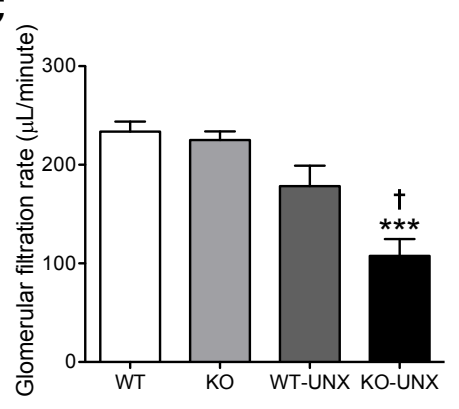

B

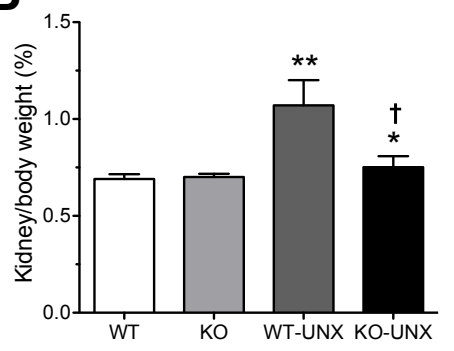

D

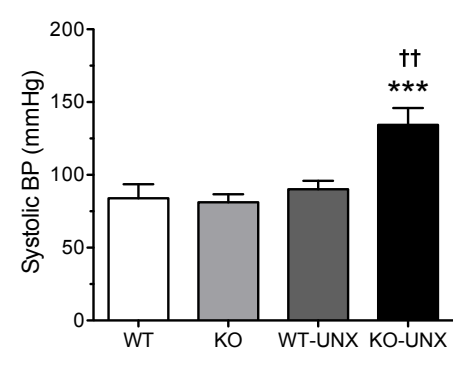

Figure 1 Body weight, kidney weight, systolic blood pressure, and glomerular filtration rate (GFR) measurements in WT-UNX and KO-UNX mice. A: Body weight of the mice that underwent uninephrectomy (WT-UNX and KO-UNX) or sham operation (WT and KO) was recorded every 2 weeks for 12 weeks after surgery. B: Kidney/body weight ratio was measured when mice were sacrificed at 12 weeks after surgery. C: GFR was measured by using the two-compartment clearance model after injection of fluorescein isothiocyanate-inulin. D: Systolic blood pressure (BP) was measured at 12 weeks after surgery by tail-cuff plethysmography. $n=6$ (A-D). ${ }^{*} P<0.05,{ }^{*} P<0.01$, and $* * * P<0.001$ versus sham-operated group; ${ }^{\dagger} P<0.05,{ }^{\dagger \dagger} P<0.01$ as compared with WT-UNX.
WT-UNX mice, suggesting a significant glomerulosclerosis in KO-UNX mice (Figure 3, A-C). A significant tubulointerstitial injury fibrosis with infiltration of inflammatory cells was also observed in KO-UNX mice, but not in WT-UNX mice (Figure 3D). Together, these findings suggest that decreased KLF2 expression in endothelial cells increases kidney injury in the setting of UNX.
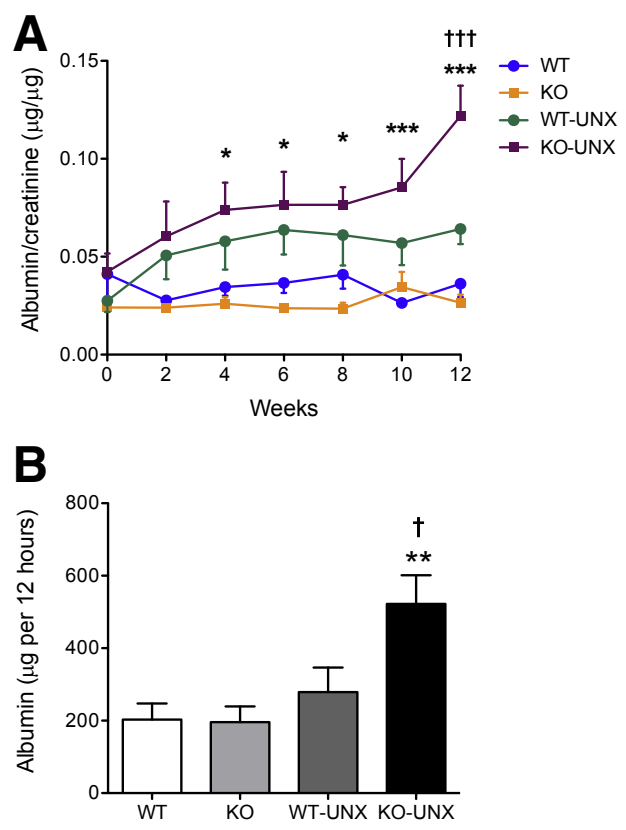

Figure 2 Urine albumin excretion rate increases in KO-UNX mice. A: Development of albuminuria was assessed by albumin/creatinine ratio in sham-operated and UNX mice. B: Twelve-hour urine albumin excretion rate was determined at 12 weeks after surgery. $n=6$ (A and $\mathbf{B})$. ${ }^{*} P<0.05$, ${ }^{* *} P<0.01$, and ${ }^{* * *} P<0.001$ versus sham-operated group; ${ }^{\dagger} P<0.05$, ${ }^{\dagger \dagger} P<0.001$ versus WT-UNX.

\section{Reduced KLF2 Expression Results in Increased Glomerular Endothelial Cell Injury after UNX}

KLF2, expressed predominantly in endothelial cells, has been described to protect endothelial cells from cellular stress. ${ }^{26}$ KLF2 has multiple binding sites in the promoter of nitric oxide synthase-3 (NOS3), ${ }^{16,27}$ which encodes endothelial nitric oxide synthase (eNOS). Previous studies suggest a critical role of eNOS in the progression of kidney disease. ${ }^{28,29}$ Endothelium-derived nitric oxide (NO) has been also shown to be involved in the compensatory renal growth in response to nephron reduction by modulating the hemodynamic changes. ${ }^{30}$ We also recently showed that eNOS expression was reduced in the diabetic Klf2 heterozygous $\mathrm{KO}$ mice as compared to diabetic WT mice. ${ }^{18}$ Similarly, we observed herein that mRNA levels of eNOS were significantly decreased in the glomeruli of KO-UNX as compared to all other groups (Figure 4A). Reduction of eNOS specifically in the KO-UNX glomeruli was further confirmed at the protein level by immunostaining (Figure 4, B and C) and by Western blot analyses (Figure 4, D-F). Significant reduction in activated eNOS (as detected by phosphorylation on Ser1176) was concomitant to a reduction in total level of eNOS.

In addition to eNOS, KLF2 also affects the expression of angiogenesis markers, such as Vegfr1, Vegfr2, Angpt1, Angpt2, and Tie-2. ${ }^{31}$ Therefore, we examined whether KLF2 reduction in endothelial cells affected their expression in the glomeruli by real-time PCR. Of the genes tested, we observed that reduced KLF2 expression in endothelial cells caused a significant increase in proangiogenic genes, namely Vegfa, Vegfr2, and Angpt2, whereas the expression of Vegfr1, Angpt1, and Tie-2 was decreased (Figure 4G). These changes are consistent with what has been reported for KLF2's function in regulating angiogenesis. ${ }^{14,31}$ 

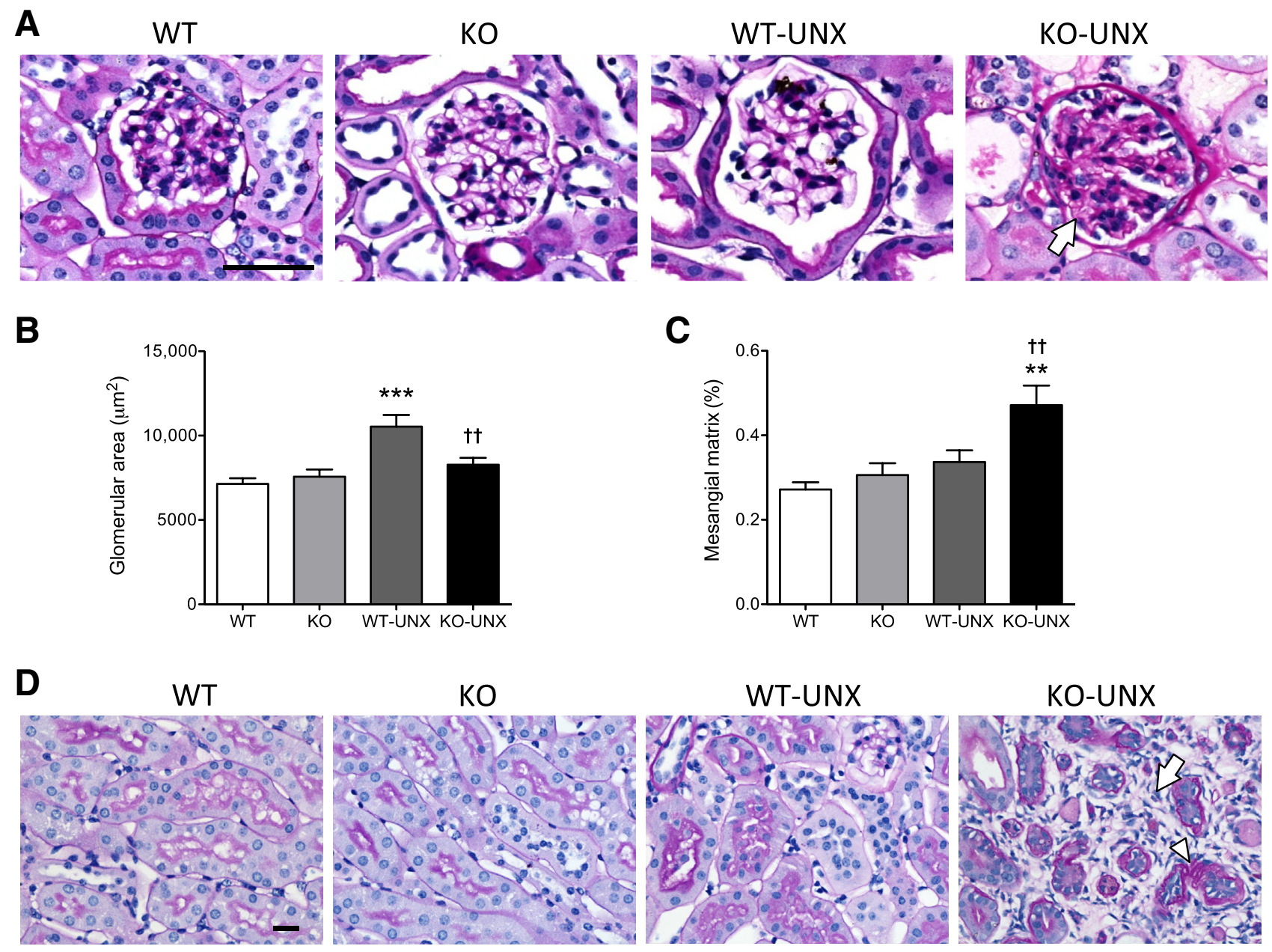

Figure 3 Kidney histology and morphometric analysis. A: Representative images of periodic acid-Schiff (PAS) staining of kidney sections from mice at 12 weeks after surgery are shown. PAS staining shows glycogen and basement membrane matrix molecules in pink and nuclei in blue. Arrow shows the area of increased mesangial matrix in K0-UNX glomeruli. Quantification of glomerular area (B) and percentage of mesangial matrix area in the glomeruli (C) are shown (60 glomeruli per group). D: Representative images of PAS staining in tubulointerstitial compartments. Arrowhead shows an area of increased tubular basement membrane (in pink) and the arrow shows an example of infiltrating cells (irregular shaped nuclei in blue) present around the tubules. $n=6$ mice (B and $\mathbf{C})$. ${ }^{* *} P<0.01,{ }^{* * *} P<0.001$ versus sham-operated groups; ${ }^{\dagger \dagger} P<0.01$ versus WT-UNX. Scale bar $=50 \mu \mathrm{m}$ (A and D). Original magnifications: $\times 400$ (A); $\times 100$ (D).

\section{Endothelial-Specific Reduction in KLF2 Results in Podocyte Injury Induced by UNX}

Because KO-UNX mice developed more albuminuria than WT-UNX mice, we speculated that they might also develop more podocyte injury than WT-UNX through the glomerular endothelial cell-podocyte cross talk. Electron microscopy of kidney sections revealed a significant increase in podocyte effacement in the KO-UNX mice compared to WT-UNX mice (Figure 5, A and B). We also assessed the podocyte number per glomerular cross section and per $1000 \mu \mathrm{m}^{2}$ of glomerular areas using a known podocyte marker, Wilms' tumor-1 (WT1). Consistent with increased foot process effacement, the number of WT-1 positive podocytes was markedly reduced in KO-UNX, but not in the WT-UNX, when compared to sham-operated mice (Figure 5, $\mathrm{C}-\mathrm{E}$ ). Decreased podocyte number in KO-UNX was further confirmed by significantly diminished mRNA expression of podocyte-specific markers (WT1, podocin, and synaptopodin) in isolated glomeruli (Figure 5F). Taken together, these data suggest that reduced KLF2 expression in endothelial cells increases the susceptibility to endothelial injury, resulting in eventual albuminuria as a result of podocyte foot process effacement and loss through the mechanism of glomerular endothelial cell-podocyte cross talk.

\section{KLF2 Expression in the Kidney Is Reduced in Nephrectomy Patients with Progressive Kidney Disease}

Finally, to ascertain whether the reduced expression level of KLF2 is associated with patients with progressive kidney disease, we selected 10 nephrectomy patients, among which five had increased serum creatinine at the follow-up laboratories in 1 and 2 years (progression group), whereas the other five had normal renal function at the follow-up (nonprogression group) (Table 2), and archival kidney tissues from these patients were used for immunostaining 


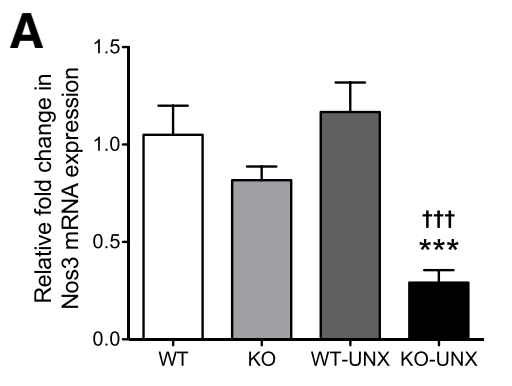

B
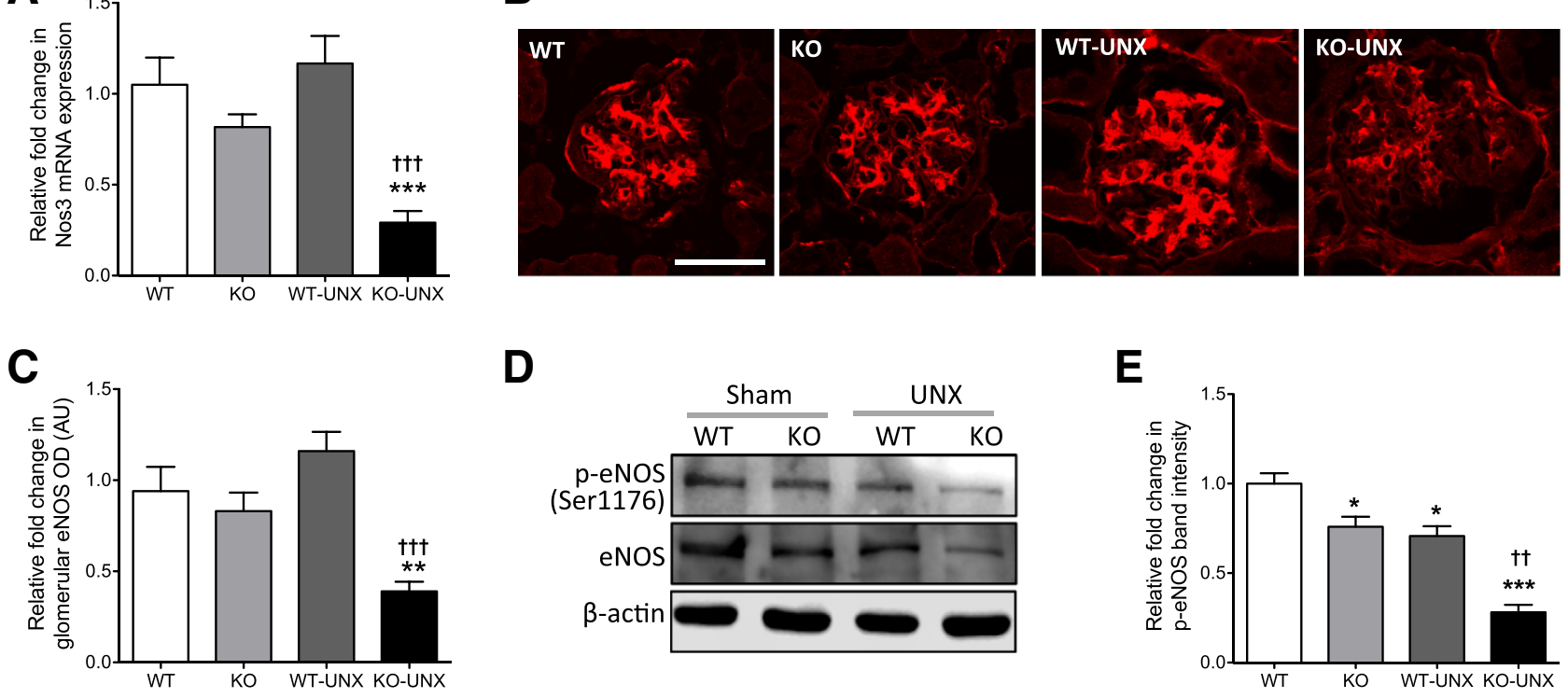

D
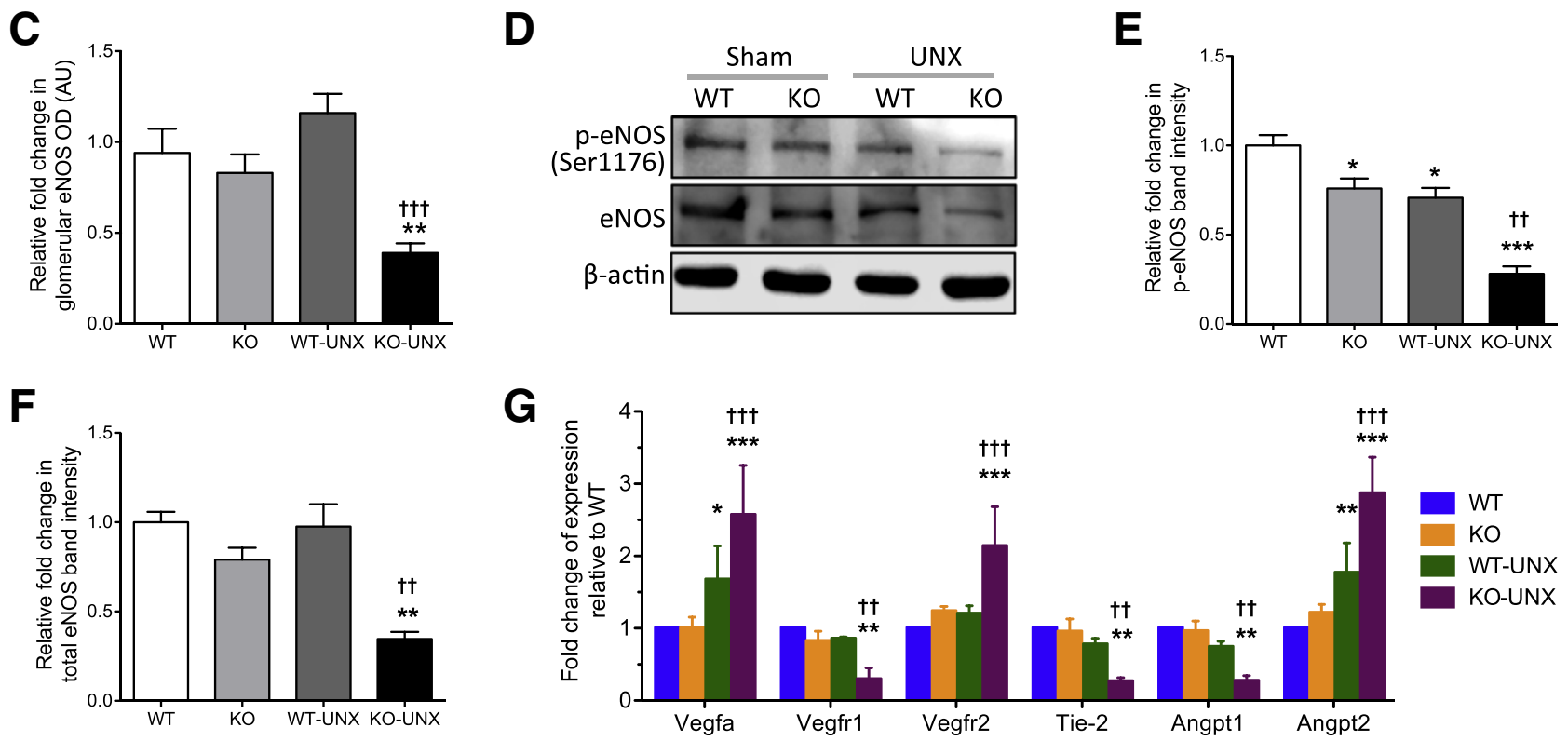

Figure 4 Expression of endothelial nitric oxide synthasae (eNOS) and glomerular endothelial cell markers. A: eNOS expression in isolated glomeruli of mice were assessed by real-time PCR. B: Representative images of immunofluorescence staining for eNOS are shown. C: Optical density (OD) immunofluorescence staining was measured and quantified as relative fold-changes to WT mice (60 glomeruli per group). D: Representative image of a Western blot of phosphorylated eNOS (p-Ser1176) and total eNOS (probed in the same blot) is shown. $\beta$-Actin is used as a loading control. E and F: Densitometric analysis of p-eNOS (E) and total eNOS (F). G: Vegfa, Vefr2, Vegfr1, Tie2, Angpt1, and Angpt2 mRNA levels were measured in the isolated glomeruli of mice by real-time PCR. $n=6(\mathbf{A}$ and $\mathbf{C}) ; n=3(\mathbf{E}-\mathbf{G}) .{ }^{*} P<0.05,{ }^{* *} P<0.01,{ }^{* * *} P<0.001$ versus sham-operated groups; ${ }^{\dagger \dagger} P<0.01,{ }^{\dagger \dagger \dagger} P<0.001$ versus WT-UNX. Scale bar $=50 \mu \mathrm{m}($ B). Original magnification, $\times 400$ (B). AU, arbitrary unit.

of KLF2 (Figure 6, A-F). As Table 2 shows, number of patients with diabetes mellitus and hypertension were unchanged from baseline to the follow-up period in both groups. Immunostaining revealed that the expression of KLF2 was significantly reduced in patients with progression of kidney disease (Figure 6, D-G) in comparison to those without progression (Figure 6, A-C and G). Although larger sample size is required for further validation, together with in vivo data in UNX mice, our findings suggest that decreased KLF2 expression is associated with the progression of human kidney disease in nephrectomy patients.

\section{Discussion}

Glomerular hyperfiltration and hypertrophy induced by nephron reduction is considered as a major event leading to the progression of kidney disease ${ }^{2}$; consequently, it is critical for us to understand the cellular and molecular mechanism(s) mediating this process. Glomerular hyperfiltration causes shear stress in endothelial cells, ${ }^{32}$ which is known to increase KLF2 expression. ${ }^{26}$ Recently, we reported that reduced KLF2 expression in endothelial cells leads to more severe glomerular endothelial cell injury and kidney damage in early diabetic nephropathy, which is characterized by glomerular hyperfiltration. ${ }^{18}$ However, we were unable to assess the relationship between hyperfiltration and KLF2 expression in diabetic mice, because KLF2 expression is also affected by high glucose and insulin. Therefore, we used the UNX model, which allows us to address the role of KLF2 in mediating glomerular hyperfiltration-induced kidney injury. In addition, this current study further validates the critical role of KLF2 in glomerular endothelial injury by using another mouse model of CKD.

Endothelial-specific reduction of KLF2 expression resulted in increased glomerular endothelial cell injury, podocyte loss, proteinuria, glomerulosclerosis, and tubulointerstitial injury 
A
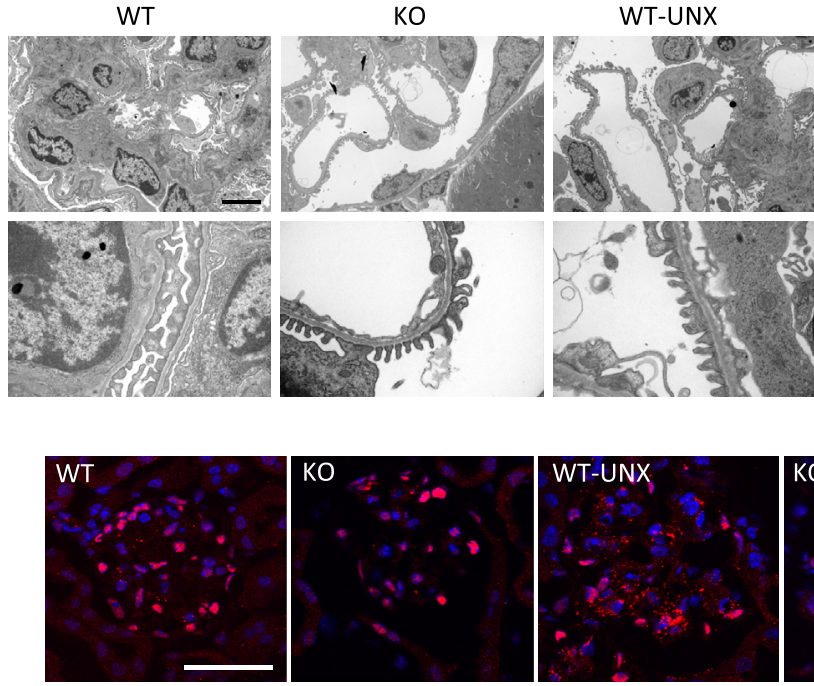

KO-UNX

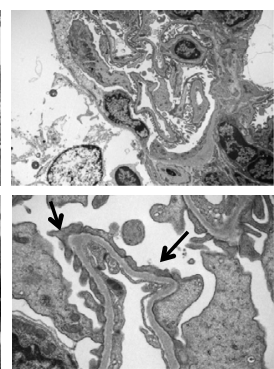

C
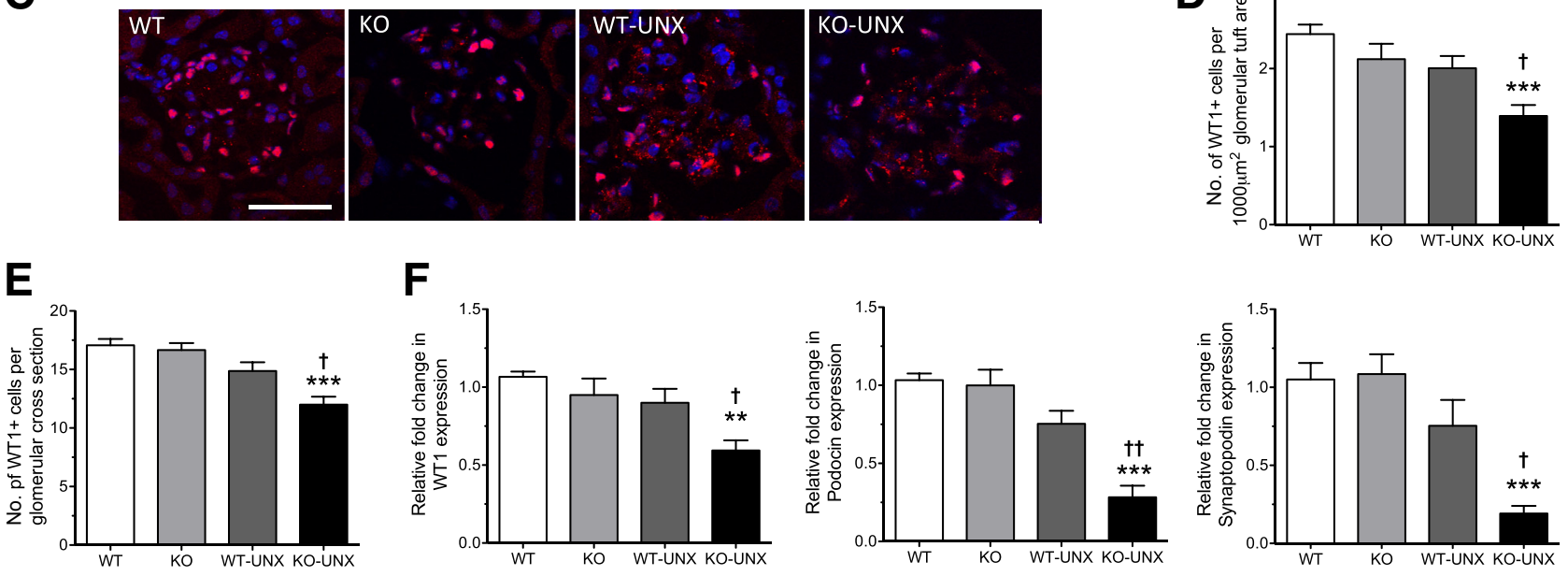

Figure 5 Podocyte foot process effacement and podocyte loss in KO-UNX glomeruli. A: Representative images of electron microscopy from the WT, KO, WT-UNX, K0-UNX mice are shown with both low and high magnifications. K0-UNX glomeruli display effacement of podocyte foot processes (arrows). B: Quantification of foot process effacement (60 glomeruli per group). C: Representative images of WT1 staining in glomeruli are shown. Number of WT1-positive cells per glomerular cross section (D) and per $1000 \mu \mathrm{m}^{2}$ glomerular tuft area (E) were quantified (60 glomeruli per group). F: mRNA levels of WT1, podocin, and synaptopodin were measured by using real-time PCR and expressed as fold-change in comparison to WT. $n=6(\mathbf{B}-\mathbf{F}) .{ }^{* *} P<0.01,{ }^{* * *} P<0.001$ versus sham-operated group; ${ }^{\dagger} P<0.05,{ }^{\dagger \dagger} P<0.01$ versus WT-UNX. Scale bars: $5 \mu \mathrm{m}(\mathbf{A}) ; 50 \mu \mathrm{m}$ (C). Original magnification: $\times 2000(\mathbf{A}$, top row); $\times 10,000$ (A, bottom row); $\times 400$ (C).

after UNX, suggesting that KLF2 protects the kidney from injury in response to nephron reduction through an endoprotective mechanism as described previously. ${ }^{26}$ This protective mechanism of KLF2 appears to be mediated in part by its regulation of eNOS expression. The enzymatic activity of eNOS is stimulated by changes in the hydromechanical forces associated with pulsatile blood flow. ${ }^{33}$ Among such mechanical forces, shear stress is a major stimulus for

Table 2 Clinical Parameters for Patients with Nephrectomy

\begin{tabular}{|c|c|c|c|c|c|c|c|c|c|}
\hline Patient no. & Sex & $\begin{array}{l}\text { Age } \\
\text { (years) }\end{array}$ & $\begin{array}{l}\text { Baseline } \\
\mathrm{Cr}(\mathrm{mg} / \mathrm{dL})\end{array}$ & $\begin{array}{l}\mathrm{F} / \mathrm{U} \mathrm{Cr} \text { year } 1 \\
(\mathrm{mg} / \mathrm{dL})\end{array}$ & $\begin{array}{l}\mathrm{F} / \mathrm{U} \text { Cr year } 2 \\
(\mathrm{mg} / \mathrm{dL})\end{array}$ & $\begin{array}{l}\text { Baseline } \\
\text { DM }\end{array}$ & $\begin{array}{l}\mathrm{F} / \mathrm{U} \mathrm{DM} \\
\text { year } 2\end{array}$ & $\begin{array}{l}\text { Baseline } \\
\text { HTN }\end{array}$ & $\begin{array}{l}\text { F/U HTN } \\
\text { year } 2\end{array}$ \\
\hline \multicolumn{10}{|l|}{ Progression } \\
\hline 1 & M & 64 & 1.1 & 1.4 & 1.4 & Yes & Yes & Yes & Yes \\
\hline 2 & $\mathrm{~F}$ & 61 & 1 & 2 & 1.5 & Yes & Yes & Yes & Yes \\
\hline 3 & M & 82 & 1.3 & 2 & 1.9 & No & No & Yes & Yes \\
\hline 4 & M & 71 & 1.1 & 1.6 & 1.7 & Yes & Yes & Yes & Yes \\
\hline 5 & M & 59 & 1.1 & 1.6 & 1.3 & No & No & No & No \\
\hline \multicolumn{10}{|c|}{ Nonprogression } \\
\hline 6 & M & 74 & 0.9 & 0.9 & 0.9 & No & No & No & No \\
\hline 7 & M & 66 & 0.8 & 0.9 & 0.9 & No & No & Yes & Yes \\
\hline 8 & $\mathrm{~F}$ & 60 & 0.9 & 0.8 & 0.8 & No & No & No & No \\
\hline 9 & $\mathrm{~F}$ & 72 & 1 & 0.8 & 1 & No & No & Yes & Yes \\
\hline 10 & M & 65 & 0.8 & 1 & 0.8 & No & No & Yes & Yes \\
\hline
\end{tabular}

All 10 patients underwent nephrectomy because of renal tumors. Serum creatinine was obtained at the baseline level before the surgery and 1 or 2 years after surgery. Patients 1 to 5 had increased serum creatinine after nephrectomy, whereas patients 6 to 10 had stable renal function after nephrectomy. The kidney samples were used for immunostaining for KLF2.

F, female; M, male; $C r$, creatinine; DM, diabetes mellitus; F/U, follow-up; HTN, hypertension. 

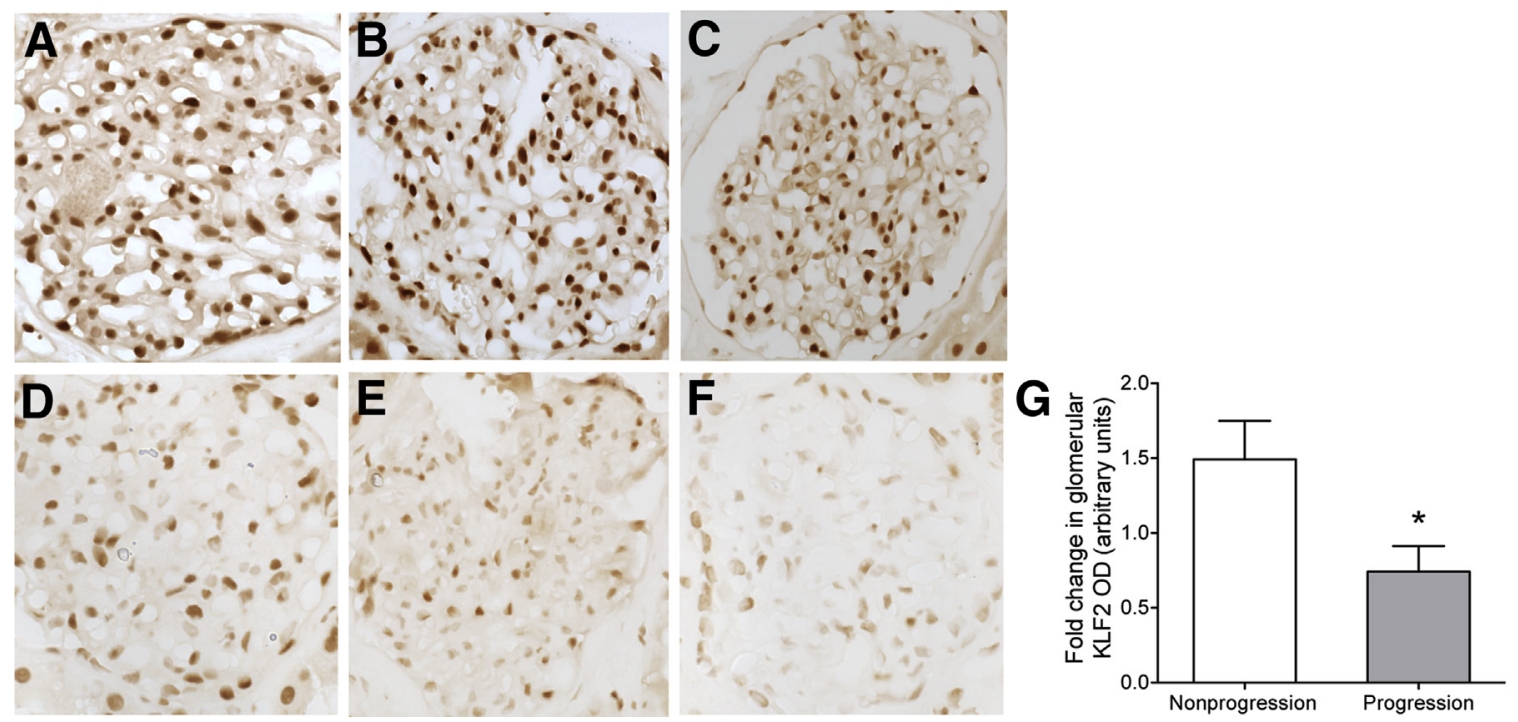

Figure 6 Krüppel-like factor 2 (KLF2) expression in human kidney tissues from patients with nephrectomy. A-F: The representative images of KLF2 immunostaining in kidneys of three nephrectomy patients with progressive kidney disease (D-F) and three with nonprogression (A-C). A total of 10 nephrectomy patients were included in which five patients had increased serum creatinine at the follow-up (progression) and five patients with normal renal function at the follow-up (nonprogression), as shown in Table 2. G: The quantification of the staining was performed and was compared between nephrectomy patients with and without progression. $n=5(\mathbf{G}) .{ }^{*} P<0.05$. Original magnification, $\times 400(\mathbf{A}-\mathbf{F})$.

endogenous NO production from the endothelium in which KLF2 plays a major role. ${ }^{26}$ Studies in various animal models demonstrated that impaired availability of NO, caused by either inhibition or genetic defect of eNOS, accelerates the progression of kidney disease. ${ }^{28,29}$ Several studies suggest that both endothelium and endothelium-derived NO play a critical role in the compensatory renal growth by modulating the hemodynamic changes ${ }^{30}$ and that increased renal synthesis of NO mediated by eNOS is the initial response to UNX necessary for the hypertrophy of the remnant kidney. $^{34,35}$

KLF2 has been described to regulate several other important genes involved in vascular endothelial cell development, differentiation, and function. ${ }^{26}$ Our studies suggest that reduced KLF2 expression in endothelial cells caused a significant increase in Vegfa, Vegfr2, and Angpt2 mRNA expression and decrease in Vegfr1, Angpt1, and Tie-2 after UNX. The alteration of expression of these genes is known to be associated with CKD, such as diabetic nephropathy. ${ }^{36}$ Although the exact mechanisms and consequences of these gene expression changes remain to be determined, it suggests that KLF2 may be up-regulated by glomerular hyperfiltration-induced shear stress and protect against endothelial cell injury through direct and indirect transcriptional regulation of several key endothelial cell markers.

Because of lack of a glomerular endothelial cell-specific Cre mouse model, herein we have used global endothelial cell-specific Cre transgenic mice to obtain the endothelial heterozygous Klf2 knockout. Therefore, the potential effects of systemic endothelial injury in KO-UNX mice cannot be excluded. The elevated blood pressure observed in KOUNX mice is likely a result of combination of reduced endothelial expression of eNOS and declining renal function because of significant glomerulosclerosis, tubular cell atrophy, and interstitial fibrosis.

Our study also demonstrates that more severe glomerular endothelial cell injury in KO-UNX mice contributed to more podocyte injury, characterized by a reduction in podocyte number and increased podocyte foot process effacement, thereby suggesting a potential cross talk between glomerular endothelial cells and podocytes. Similarly, our previous studies suggest such a cross talk also exists in early diabetic nephropathy. ${ }^{18}$ Consistent with our findings, the previous study suggests that eNOS could be a key mediator of glomerular endothelial cells-podocytes cross talk. ${ }^{37}$ However, the precise mechanism(s) mediating this cross talk will be the focus of future studies. Interestingly, KO-UNX mice developed more mesangial expansion as compared to the other groups, suggesting a potential cross talk between glomerular endothelial cells and mesangial cells as well.

Our human data on KLF2 expression suggest that decreased KLF2 expression is associated with the progression of kidney disease in patients who underwent nephrectomy. However, because our study was limited to a small number of available patient samples, we cannot rule out other confounding factors (eg, diabetes mellitus and hypertension) that may affect the progression of kidney disease in these patients. In addition, although normal tissues were obtained from patients with renal tumors at a good margin, we cannot completely rule out the effects of tumors in the surrounding normal tissue, although both progressive and nonprogressive groups are from patients with renal tumors and the potential effects of tumors on KLF2 expression would be similar in both groups. 
Therefore, an independent study using larger patient cohort to stratify the change in KLF2 expression for potential confounders is required to confirm these initial findings in the future.

In conclusion, our data suggest a protective role of KLF2 in glomerular endothelial cell injury induced by glomerular hyperfiltration in response to nephron reduction in the UNX model. Reduction of KLF2 expression in endothelial cells leads to increased endothelial cell injury and to progressive kidney disease. Understanding the disease mechanisms mediated by glomerular hyperfiltration at early stage of CKD may help us to shed light on novel preventive strategies against the disease development.

\section{Acknowledgments}

We thank Dr. Peter Mundel for providing rabbit antipodocin antibody.

F.Z., C.E., M.M., F.S., and S.K.M. performed experiments; J.M. provided Klf2 floxed mice; and J.C.H., K.L., S.K.M., F.Z., H.Y.C., and Y.J.W. designed experiments, analyzed the data, and wrote the paper. J.H. is the guarantor of this work and, as such, had full access to all of the data in the study and takes responsibility for the integrity of the data and the accuracy of the data analysis.

\section{References}

1. Coresh J, Selvin E, Stevens LA, Manzi J, Kusek JW, Eggers P, Van Lente F, Levey AS: Prevalence of chronic kidney disease in the United States. JAMA 2007, 298:2038-2047

2. Brenner BM, Lawler EV, Mackenzie HS: The hyperfiltration theory: a paradigm shift in nephrology. Kidney Int 1996, 49:1774-1777

3. Feinberg MW, Lin Z, Fisch S, Jain MK: An emerging role for Kruppel-like factors in vascular biology. Trends Cardiovasc Med 2004, 14:241-246

4. Pearson R, Fleetwood J, Eaton S, Crossley M, Bao S: Kruppel-like transcription factors: a functional family. Int J Biochem Cell Biol 2008, 40:1996-2001

5. Kuo CT, Veselits ML, Barton KP, Lu MM, Clendenin C, Leiden JM: The LKLF transcription factor is required for normal tunica media formation and blood vessel stabilization during murine embryogenesis. Genes Dev 1997, 11:2996-3006

6. Carlson CM, Endrizzi BT, Wu J, Ding X, Weinreich MA, Walsh ER, Wani MA, Lingrel JB, Hogquist KA, Jameson SC: Kruppel-like factor 2 regulates thymocyte and T-cell migration. Nature 2006, 442:299-302

7. Das H, Kumar A, Lin Z, Patino WD, Hwang PM, Feinberg MW, Majumder PK, Jain MK: Kruppel-like factor 2 (KLF2) regulates proinflammatory activation of monocytes. Proc Natl Acad Sci U S A 2006, 103:6653-6658

8. Weinreich MA, Takada K, Skon C, Reiner SL, Jameson SC, Hogquist KA: KLF2 transcription-factor deficiency in T cells results in unrestrained cytokine production and upregulation of bystander chemokine receptors. Immunity 2009, 31:122-130

9. Mallipattu SK, Liu R, Zheng F, Narla G, Ma'ayan A, Dikman S, Jain MK, Saleem M, D'Agati V, Klotman P, Chuang PY, He JC: Kruppel-like factor 15 (KLF15) is a key regulator of podocyte differentiation. J Biol Chem 2012, 287:19122-19135

10. Lee MJ, Feliers D, Mariappan MM, Sataranatarajan K, Mahimainathan L, Musi N, Foretz M, Viollet B, Weinberg JM,
Choudhury GG, Kasinath BS: A role for AMP-activated protein kinase in diabetes-induced renal hypertrophy. Am J Physiol Renal Physiol 2007, 292:F617-F627

11. Parmar KM, Larman HB, Dai G, Zhang Y, Wang ET, Moorthy SN, Kratz JR, Lin Z, Jain MK, Gimbrone MA Jr, Garcia-Cardena G Integration of flow-dependent endothelial phenotypes by Kruppel-like factor 2. J Clin Invest 2006, 116:49-58

12. Lin Z, Natesan V, Shi H, Dong F, Kawanami D, Mahabeleshwar GH, Atkins GB, Nayak L, Cui Y, Finigan JH, Jain MK: Kruppel-like factor 2 regulates endothelial barrier function. Arterioscler Thromb Vasc Biol 2010, 30:1952-1959

13. Chiplunkar AR, Curtis BC, Eades GL, Kane MS, Fox SJ, Haar JL, Lloyd JA: The Kruppel-like factor 2 and Kruppel-like factor 4 genes interact to maintain endothelial integrity in mouse embryonic vasculogenesis. BMC Dev Biol 2013, 13:40

14. Bhattacharya R, Senbanerjee S, Lin Z, Mir S, Hamik A, Wang P, Mukherjee P, Mukhopadhyay D, Jain MK: Inhibition of vascular permeability factor/vascular endothelial growth factor-mediated angiogenesis by the Kruppel-like factor KLF2. J Biol Chem 2005, 280:28848-28851

15. Lin Z, Kumar A, SenBanerjee S, Staniszewski K, Parmar K, Vaughan DE, Gimbrone MA Jr, Balasubramanian V, GarciaCardena G, Jain MK: Kruppel-like factor 2 (KLF2) regulates endothelial thrombotic function. Circ Res 2005, 96:e48-e57

16. SenBanerjee S, Lin Z, Atkins GB, Greif DM, Rao RM, Kumar A, Feinberg MW, Chen Z, Simon DI, Luscinskas FW, Michel TM, Gimbrone MA Jr, Garcia-Cardena G, Jain MK: KLF2 is a novel transcriptional regulator of endothelial proinflammatory activation. $\mathrm{J}$ Exp Med 2004, 199:1305-1315

17. Slater SC, Ramnath RD, Uttridge K, Saleem MA, Cahill PA, Mathieson PW, Welsh GI, Satchell SC: Chronic exposure to laminar shear stress induces Kruppel-like factor 2 in glomerular endothelial cells and modulates interactions with co-cultured podocytes. Int $\mathrm{J}$ Biochem Cell Biol 2012, 44:1482-1490

18. Zhong F, Chen H, Wei C, Zhang W, Li Z, Jain MK, Chuang PY, Wang Y, Mallipattu SK, He JC: Reduced Kruppel-like factor 2 expression may aggravate the endothelial injury of diabetic nephropathy. Kidney Int 2015, 87:382-395

19. Kume S, Uzu T, Araki S, Sugimoto T, Isshiki K, Chin-Kanasaki M, Sakaguchi M, Kubota N, Terauchi Y, Kadowaki T, Haneda M, Kashiwagi A, Koya D: Role of altered renal lipid metabolism in the development of renal injury induced by a high-fat diet. J Am Soc Nephrol 2007, 18:2715-2723

20. Rieg T: A high-throughput method for measurement of glomerular filtration rate in conscious mice. J Vis Exp 2013:e50330

21. Takemoto M, Asker N, Gerhardt H, Lundkvist A, Johansson BR, Saito Y, Betsholtz C: A new method for large scale isolation of kidney glomeruli from mice. Am J Pathol 2002, 161:799-805

22. Potapova TA, Sivakumar S, Flynn JN, Li R, Gorbsky GJ: Mitotic progression becomes irreversible in prometaphase and collapses when Wee1 and Cdc25 are inhibited. Mol Biol Cell 2011, 22:1191-1206

23. Gassmann M, Grenacher B, Rohde B, Vogel J: Quantifying Western blots: pitfalls of densitometry. Electrophoresis 2009, 30:1845-1855

24. Koop K, Eikmans M, Baelde HJ, Kawachi H, De Heer E, Paul LC, Bruijn JA: Expression of podocyte-associated molecules in acquired human kidney diseases. J Am Soc Nephrol 2003, 14:2063-2071

25. Lee JS, Yu Q, Shin JT, Sebzda E, Bertozzi C, Chen M, Mericko P, Stadtfeld M, Zhou D, Cheng L, Graf T, MacRae CA, Lepore JJ, Lo CW, Kahn ML: Klf2 is an essential regulator of vascular hemodynamic forces in vivo. Dev Cell 2006, 11:845-857

26. Atkins GB, Jain MK: Role of Kruppel-like transcription factors in endothelial biology. Circ Res 2007, 100:1686-1695

27. Kwon IS, Wang W, Xu S, Jin ZG: Histone deacetylase 5 interacts with Kruppel-like factor 2 and inhibits its transcriptional activity in endothelium. Cardiovasc Res 2014, 104:127-137

28. Kobayashi T, Terada Y, Kuwana H, Tanaka H, Okado T, Kuwahara M, Tohda S, Sakano S, Sasaki S: Expression and function of 
the Delta-1/Notch-2/Hes-1 pathway during experimental acute kidney injury. Kidney Int 2008, 73:1240-1250

29. Satoh M, Haruna Y, Fujimoto S, Sasaki T, Kashihara N: Telmisartan improves endothelial dysfunction and renal autoregulation in Dahl salt-sensitive rats. Hypertens Res 2010, 33:135-142

30. Sigmon DH, Gonzalez-Feldman E, Cavasin MA, Potter DL, Beierwaltes WH: Role of nitric oxide in the renal hemodynamic response to unilateral nephrectomy. J Am Soc Nephrol 2004, 15: $1413-1420$

31. Dekker RJ, Boon RA, Rondaij MG, Kragt A, Volger OL, Elderkamp YW, Meijers JC, Voorberg J, Pannekoek H, Horrevoets AJ: KLF2 provokes a gene expression pattern that establishes functional quiescent differentiation of the endothelium. Blood 2006, 107: $4354-4363$

32. Srivastava T, Alon US, Cudmore PA, Tarakji B, Kats A, Garola RE, Duncan RS, McCarthy ET, Sharma R, Johnson ML, Bonewald LF, El-Meanawy A, Savin VJ, Sharma M: Cyclooxygenase-2, prostaglandin E2, and prostanoid receptor EP2 in fluid flow shear stressmediated injury in the solitary kidney. Am J Physiol Renal Physiol 2014, 307:F1323-F1333
33. Pechanova O, Rezzani R, Babal P, Bernatova I, Andriantsitohaina R: Beneficial effects of Provinols: cardiovascular system and kidney. Physiol Res 2006, 55(Suppl 1):S17-S30

34. Ozeki M, Nagasu H, Satoh M, Namikoshi T, Haruna Y, Tomita N, Sasaki T, Kashihara N: Reactive oxygen species mediate compensatory glomerular hypertrophy in rat uninephrectomized kidney. J Physiol Sci 2009, 59:397-404

35. Valdivielso JM, Perez-Barriocanal F, Garcia-Estan J, LopezNovoa JM: Role of nitric oxide in the early renal hemodynamic response after unilateral nephrectomy. Am J Physiol 1999, 276: R1718-R1723

36. Jeansson M, Gawlik A, Anderson G, Li C, Kerjaschki D, Henkelman M, Quaggin SE: Angiopoietin-1 is essential in mouse vasculature during development and in response to injury. J Clin Invest 2011, 121:2278-2289

37. Yuen DA, Stead BE, Zhang Y, White KE, Kabir MG, Thai K, Advani SL, Connelly KA, Takano T, Zhu L, Cox AJ, Kelly DJ, Gibson IW, Takahashi T, Harris RC, Advani A: eNOS deficiency predisposes podocytes to injury in diabetes. J Am Soc Nephrol 2012, 23:1810-1823 